\title{
MIMO Channel Model with Propagation Mechanism and the Properties of Correlation and Eigenvalue in Mobile Environments
}

\author{
Yuuki Kanemiyo, Youhei Tsukamoto, Hiroaki Nakabayashi, and Shigeru Kozono \\ Department of Electrical, Electronics and Computer Engineering, Chiba Institute of Technology, 2-17-1 Tsudanuma, \\ Narashino-shi, Chiba 275-0016, Japan \\ Correspondence should be addressed to Shigeru Kozono, kozono.shigeru@it-chiba.ac.jp
}

Received 29 November 2011; Revised 27 March 2012; Accepted 27 March 2012

Academic Editor: David A. Sanchez-Hernandez

Copyright ( 92012 Yuuki Kanemiyo et al. This is an open access article distributed under the Creative Commons Attribution License, which permits unrestricted use, distribution, and reproduction in any medium, provided the original work is properly cited.

\begin{abstract}
This paper described a spatial correlation and eigenvalue in a multiple-input multiple-output (MIMO) channel. A MIMO channel model with a multipath propagation mechanism was proposed and showed the channel matrix. The spatial correlation coefficient formula $\rho_{i-j, i^{\prime}-j^{\prime}}(\mathrm{bm})$ between MIMO channel matrix elements was derived for the model and was expressed as a directive wave term added to the product of mobile site correlation $\rho_{i-i^{\prime}}(m)$ and base site correlation $\rho_{j-j^{\prime}}(b)$ without LOS path, which are calculated independently of each other. By using $\rho_{i-j, i^{\prime}-j^{\prime}}(\mathrm{bm})$, it is possible to create the channel matrix element with a fixed correlation value estimated by $\rho_{i-j, i^{\prime}-j^{\prime}}(\mathrm{bm})$ for a given multipath condition and a given antenna configuration. Furthermore, the correlation and the channel matrix eigenvalue were simulated, and the simulated and theoretical correlation values agreed well. The simulated eigenvalue showed that the average of the first eigenvalue $\lambda_{1}$ hardly depends on the correlation $\rho_{i-j, i^{\prime}-j^{\prime}}(b m)$, but the others do depend on $\rho_{i-j, i^{\prime}-j^{\prime}}(\mathrm{bm})$ and approach $\lambda_{1}$ as $\rho_{i-j, i^{\prime}-j^{\prime}}(\mathrm{bm})$ decreases. Moreover, as the path moves into LOS, the $\lambda_{1}$ state with mobile movement becomes more stable than the $\lambda_{1}$ of NLOS path.
\end{abstract}

\section{Introduction}

To support realtime multimedia communication, future mobile communications will require a high-bit-rate transmission system with high utilization of the frequency spectrum in multipath channels with line-of-sight (LOS) and non-line-of-sight (NLOS) paths [1]. Systems capable of fulfilling this requirement, with features such as multiple-input multiple-output (MIMO) $[2,3]$ and orthogonal frequency division multiplexing (OFDM) $[4,5]$, have been studied extensively. MIMO is especially advantageous in high utilization of the frequency spectrum. In MIMO, transmission quality and capacity depend on the channel matrix, which consists of the complex transmission coefficients between MIMO antenna elements at a mobile terminal and at the base station. The channel matrix seems to be evaluated by the spatial correlation between matrix elements and the matrix eigenvalue, for which low correlation and a large eigenvalue are better [6]. The source of those properties is in the
MIMO channel model composed of multipath propagation and the conditions at the mobile and base sites, and many models have been proposed and studied analytically and experimentally [7-10]. As an analytical model that tries to produce the matrix with a given fixed spatial correlation between MIMO antenna elements, the stochastic MIMO channel model was analyzed on the basis of an independent and identically distributed (i.i.d) random matrix for oneside correlations at the mobile and base sites, and it has also been verified experimentally $[11,12]$. However, the analysis method used in the model seems to have trouble interpreting the channel situation directly and visualizing the physical propagation in MIMO transmission studies. Moreover, most models proposed to date have been stationary and NLOS, and there has been little analytical work on the correlation between both sides and LOS, which is still being developed.

With this in mind, we proposed a MIMO channel model with a propagation mechanism composed of multipath propagation and mobile- and base-site antenna 
configurations and created the channel matrix on the basis of the model. The channel matrix is allowed to consist of matrix elements with a given fixed theoretical correlation between antenna elements at the mobile and base sites because the propagation mechanism is known and we can calculate the correlation. Therefore, the matrix requires a formula for estimating the correlation between each pair of antenna elements at one side and at both sides under various multipath conditions and base- and mobile-site situations. So we derived the correlation formulas by using the matrix for indoors, outdoors, and so forth. Using the matrix, we could also calculate the matrix eigenvalue and we clarified its properties by simulation; moreover, it was possible to study the relation between the correlation and eigenvalue.

This paper is organized as follows. Section 2 covers the theoretical study. First, we describe the MIMO channel model with the propagation mechanism and antenna configurations and then show the channel matrix on the basis of the model. Next, we derive the correlation formulas between antenna elements at one side and between both sides in various site conditions and environments. Section 3 covers simulation. The simulation was done for the correlation and channel matrix eigenvalue with various parameter settings. The simulated and theoretical correlations are discussed and the eigenvalue's properties are described; moreover, the relation between the correlation and the eigenvalue is studied. Finally, Section 4 summarizes the results.

\section{Theory}

2.1. MIMO Channel Model. MIMO systems will be used in various areas: the cells are called pico, micro, and macro cells. The MIMO channel model, which consists of a delay profile measured around the base- and mobile-site origins and the antenna configurations with coordinate systems common to the profile's angle, is shown in Figure 1. The coordinate systems have the origin at the first antenna element center with $i=1$ for mobile site and $j=1$ for base site, respectively. The delay profile has both horizontal azimuth angles $\left(\xi_{n}, \zeta_{n}\right)$ incident to multipath scattering and to the receiving point [13-15], except for path data with an ordinary delay profile. Moreover, each arriving wave expresses a representative wave, which is the peak value in each cluster. The delay profile assumed the following conditions.

(i) The number of arriving waves is $N+1$, waves are independent of each other, and the $n$ th-path wave is denoted by subscript $n$, where $n=0$ means a directive wave and $n \geq 1$ means no directive waves.

(ii) The waves have excess delay time $\tau_{n}$ relative to the shortest path between the two origins and maximum excess delay time $\tau_{\max }$. The $\tau_{n}$ values are random over range $0 \leq \tau_{n} \leq \tau_{\max }$.

(iii) The amplitude is $h_{n}$ and independent of $\xi_{n}$ and $\zeta_{n}$, the power ratio of the directive and nondirective waves is denoted by $k$ ( $K$ in dB: Rice factor $k)$, and $k=0(K=$ $-\infty \mathrm{dB})$ means NLOS. Furthermore, the nondirective wave's power is normalized to 1 .

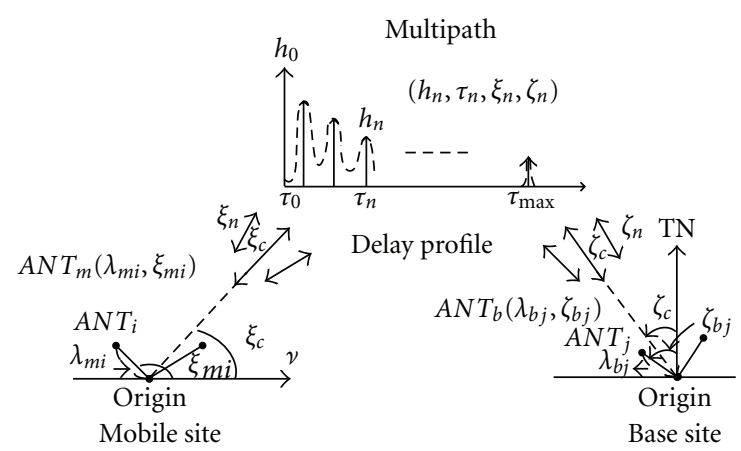

FIgURe 1: MIMO channel model.

(iv) Whether a wave's arriving angle at the mobile or base is also the incident angle to multipath scattering from the mobile or base site depends on whether the site is receiving or transmitting. Here, the mobile-site angle is denoted by $\xi_{n}$ and the base-site angle is denoted by $\zeta_{n}$. The $\xi_{n}$ and $\zeta_{n}$ are counterclockwise angles from TN (true north) at the origins on both sites; however, when the mobile station moves, $\xi_{n}$ is the angle from the mobile's movement direction. The arriving wave's initial phase is $\phi_{n}$ and the values are random over $0 \leq$ $\phi_{n}<2 \pi$.

On the other hand, assuming that all the antennas used have the same pattern with omnidirectionality and no mutual coupling, the antenna coordinates use a polar coordinate system centered at each site's origin, as shown in Figure 1. For the base site, the coordinates are denoted by $\operatorname{ANT}_{b}\left(\lambda_{b j}, \zeta_{b j}\right)$, where $\lambda_{b j}$ and $\zeta_{b j}$ mean the radius normalized by wavelength $\lambda$ and counterclockwise angle from TN for the $j$ th antenna, respectively. For a mobile site, the coordinates are similarly denoted by $\operatorname{ANT}_{m}\left(\lambda_{m i}, \xi_{m i}\right)$; however, when the mobile station moves, $\xi_{m i}$ is also the angle from the mobile's movement direction.

In this paper, we also assume that all antenna elements of each station have the same values of $\xi_{n}$ and $\zeta_{n}$, but strictly $\xi_{n}$ and $\zeta_{n}$ differ slightly among the elements by value $\delta$. The angular difference $\delta$ from $\xi_{n}$ or $\zeta_{n}$ for the normalized distance $(r / \lambda)$ between the origin and multipath scattering when the antenna element is set at spacing $\lambda$ away from the origin is shown in Figure 2. This $\delta$ is less than $0.01 \mathrm{rad}$ when $r / \lambda=100$. As shown later, the spatial correlation is sensitive to $\xi_{n}$ or $\zeta_{n}$ when the antenna is high and far away and when $\xi_{n}$ and $\zeta_{n}$ have Gaussian distributions, but not so sensitive to $\xi_{n}$ or $\zeta_{n}$ when $r / \lambda$ is small and $\xi_{n}$ and $\zeta_{n}$ are spread widely, as in the case with mobile stations or indoor cells.

2.2. MIMO Channel Matrix. Under the conditions described above and assuming a narrowband system such as OFDM, the MIMO channel, which is composed of the $j$ th base- and $i$ th mobile-station antenna elements, is denoted by MIMO 


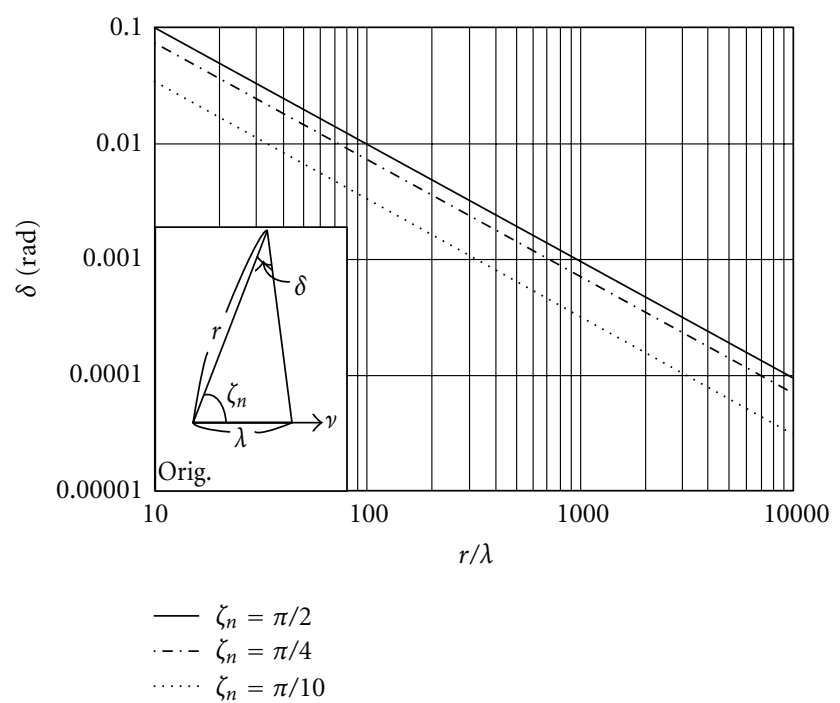

Figure 2: Angle difference $\delta$ from $\xi_{n}$ or $\zeta_{n}$ (for space $\lambda$ from origin).

$\operatorname{ch}(i-j)$, and the complex received signal level $E_{i-j}\left(t, f_{c}\right)$ is given by

$$
\begin{gathered}
E_{i-j}\left(t, f_{c}\right)=\sum_{n=0}^{N} h_{n} e^{j \theta_{i-j, n}}, \\
\theta_{i-j, n}=2 \pi\left[f_{c} \tau_{n}-f_{m} t \cos \xi_{n}-\lambda_{m i} \cos \left(\xi_{n}-\xi_{m i}\right)\right. \\
\left.-\lambda_{b j} \cos \left(\zeta_{n}-\zeta_{b j}\right)\right]+\phi_{n},
\end{gathered}
$$

where $\theta_{i-j, n}$ is the path phase of MIMO $\operatorname{ch}(i-j)$ for the $n$th multipath wave, $f_{c}$ is the radio frequency, and $f_{m}$ is the maximum Doppler frequency. The third and fourth terms of $\theta_{i-j, n}$ in brackets depend on the mobile- and basestation antenna configurations and mean phase difference from mobile and base origins, respectively.

When the number of antenna elements at each station is $M$, the MIMO channel matrix $E$, which describes the connection between both stations, can be expressed as

$$
E=\left[\begin{array}{cccc}
E_{1-1} & E_{1-2} & \cdots & E_{1-M} \\
E_{2-1} & E_{2-2} & \cdots & E_{2-M} \\
\cdot & \cdot & \cdots & \cdot \\
E_{M-1} & E_{M-2} & \cdots & E_{M-M}
\end{array}\right],
$$

where $E_{i-j}$ means $E_{i-j}\left(t, f_{c}\right)$ in (1).

\subsection{Correlation between MIMO Channel Matrix Elements}

2.3.1. General Formula. We start by studying the general spatial correlation between MIMO $\operatorname{ch}(i-j)$ and $\operatorname{ch}\left(i^{\prime}-j^{\prime}\right)$; the complex correlation is denoted by $\rho_{i-j, i^{\prime}-j^{\prime}}(b m)$. With variables $x$ meaning $E_{i-j}\left(t, f_{c}\right)$ and $y$ meaning $E_{i^{\prime}-j^{\prime}}\left(t, f_{c}\right)$ obtained by $(1), \rho_{i-j, i^{\prime}-j^{\prime}}(\mathrm{bm})$ is expressed by

$$
\begin{aligned}
& \rho_{i-j, i^{\prime}-j^{\prime}}(b m) \\
& \quad=\frac{\left\langle(x-\langle x\rangle)^{*}(y-\langle y\rangle)\right\rangle}{\left[\left\langle(x-\langle x\rangle)^{*}(x-\langle x\rangle)\right\rangle\left\langle(y-\langle y\rangle)^{*}(y-\langle y\rangle)\right\rangle\right]^{1 / 2}} .
\end{aligned}
$$

Here, the symbols \langle\rangle and $*$ mean ensemble average and conjugate complex, respectively. Under the conditions in Section $2.1,\langle x\rangle$ and $\langle y\rangle$ are zero owing to the independence of $h_{n}$ and $\theta_{i-j, n}\left(\right.$ or $\theta_{i^{\prime}-j^{\prime}, n}$ ) with random values from 0 to $2 \pi$. Therefore, the denominator in (4) is $k+1$, that is, received power. On the other hand, $\left\langle(x-\langle x\rangle)^{*}(y-\langle y\rangle)\right\rangle$ in the numerator of (4) is expressed by (5), where $\Delta \theta_{0}$ expresses the directive wave's path phase difference between MIMO $\operatorname{ch}\left(i^{\prime}-j^{\prime}\right)$ and $\operatorname{ch}(i-j)$ (see Appendix A).

$$
\begin{gathered}
\left\langle(x-\langle x\rangle)^{*}(y-\langle y\rangle)\right\rangle \\
=\left\langle h_{0}^{2} \exp \left(j \Delta \theta_{0}\right)\right\rangle \\
+\left\langle\sum_{n=1}^{N} h_{n}^{2}\right\rangle\left\langle\sum _ { n = 1 } ^ { N } \left[\cos \left(2 \pi z_{i-i^{\prime}} \cos \left(\xi_{n}-\psi_{i-i^{\prime}}\right)\right)\right.\right. \\
\left.\left.-j \sin \left(2 \pi z_{i-i^{\prime}} \cos \left(\xi_{n}-\psi_{i-i^{\prime}}\right)\right)\right]\right\rangle \\
\times\left\langle\sum _ { n = 1 } ^ { N } \left[\cos \left(2 \pi z_{j-j^{\prime}} \cos \left(\zeta_{n}-\psi_{j-j^{\prime}}\right)\right)\right.\right. \\
\left.\left.-j \sin \left(2 \pi z_{j-j^{\prime}} \cos \left(\zeta_{n}-\psi_{j-j^{\prime}}\right)\right)\right]\right\rangle \\
=k \exp \left(j \Delta \theta_{0}\right)+\rho_{i-i^{\prime}}(m) \cdot \rho_{j-j^{\prime}}(b) .
\end{gathered}
$$

Here, $z_{i-i^{\prime}}, \Psi_{i-i^{\prime}}$, and $z_{j-j^{\prime}}, \Psi_{j-j^{\prime}}$ in (5) are antenna construction parameters for the mobile and base sites, respectively. The $z_{i-i^{\prime}}$ and $\Psi_{i-i^{\prime}}$ are given by (7) and mean, respectively, the spacing between the $i$ th and $i^{\prime}$ th antenna elements and the counterclockwise angle from $\mathrm{TN}$ or the movement direction to a line with both these elements.

$$
\begin{aligned}
z_{i-i^{\prime}} & =\left[a^{2}+b^{2}\right]^{1 / 2}, \quad \psi_{i-i^{\prime}}=\tan ^{-1}\left(\frac{b}{a}\right) \\
a & =\lambda_{m i^{\prime}} \cos \xi_{m i^{\prime}}-\lambda_{m i} \cos \xi_{m i}, \\
b & =\lambda_{m i^{\prime}} \sin \xi_{m i^{\prime}}-\lambda_{m i} \sin \xi_{m i} .
\end{aligned}
$$

The first and second terms in (5) are concerned with directive and nondirective waves. Moreover, three ensemble averages in the second term are nondirective wave power, that is, 1, mobile and base station factors, which are calculated independently of each other. Therefore, we denote them as 
shown $\rho_{i-i^{\prime}}(m)$ and $\rho_{j-j^{\prime}}(b)$ in (6). Furthermore, we expand $\rho_{i-i^{\prime}}(m)$ and $\rho_{j-j^{\prime}}(b)$ to a Neumann expansion because the ensemble averages are integrated with respect to $\xi_{n}$ and $\zeta_{n}$ [16], and we get (9) for the mobile site (see Appendix B).

$$
\begin{gathered}
\rho_{i-i^{\prime}}(m) \\
=\left\langle\sum_{n=1}^{N}\left[\sum_{l=0}^{\infty} \varepsilon_{l}(-1)^{l} J_{2 l}\left(2 \pi z_{i-i^{\prime}}\right) \cos \left(2 l\left(\xi_{n}-\psi_{i-i^{\prime}}\right)\right)\right]\right\rangle \\
-j\left\langle\sum _ { n = 1 } ^ { N } \left[ 2 \sum_{l=0}^{\infty}(-1)^{l} J_{2 l+1}\left(2 \pi z_{i-i^{\prime}}\right) \cos ((2 l+1)\right.\right. \\
\left.\left.\left.\times\left(\xi_{n}-\psi_{i-i^{\prime}}\right)\right)\right]\right\rangle .
\end{gathered}
$$

Here, $\varepsilon_{l}=1(l=0), \varepsilon_{l}=2(l \geq 1), J_{2 l}(\cdot)$ is Bessel function of the first order. We can also get $\rho_{j-j^{\prime}}(b)$ for the base site in a similar manner to that for $\rho_{i-i^{\prime}}(m)$. From the above description, we can get finally the general formula for MIMO channel spatial correlation $\rho_{i-j, i^{\prime}-j^{\prime}}(\mathrm{bm})$ by rewriting (4) as

$$
\rho_{i-j, i^{\prime}-j^{\prime}}(b m)=\frac{k \exp \left(j \Delta \theta_{0}\right)+\rho_{i-i^{\prime}}(m) \cdot \rho_{j-j^{\prime}}(b)}{k+1},
$$

where $\rho_{i-i^{\prime}}(m)$ and $\rho_{j-j^{\prime}}(b)$ are mobile and base site correlations without a directive wave. Though the value of the numerator in (10) depends strongly on the first term, that is, $k$ and $\Delta \theta_{0}$, it becomes $\rho_{i-i^{\prime}}(m) \rho_{j-j^{\prime}}(b)$ without LOS, so $\rho_{i-j, i^{\prime}-j^{\prime}}(b m)$ is the product of $\rho_{i-i^{\prime}}(m)$ and $\rho_{j-j^{\prime}}(b)$. Moreover, (10) shows that $\rho_{i-j, i^{\prime}-j^{\prime}}(\mathrm{bm})$ is calculated from three items: the mobile- and base-site antenna configurations $\left(z_{i-i^{\prime}}, z_{j-j^{\prime}}, \Psi_{i-i^{\prime}}, \Psi_{j-j^{\prime}}\right)$, the angle distribution for arriving and incident waves to multiple paths $\left(\xi_{n}, \zeta_{n}\right)$, and Rice factor $k$. By using (10), we can calculate $\rho_{i-j, i^{\prime}-j^{\prime}}(\mathrm{bm})$ for any MIMO channel matrix since $i, i^{\prime}, j$, and $j^{\prime}$ can be chosen freely within $M$. Here $i=i^{\prime}$ and $j=j^{\prime}$ mean $\rho_{i-i^{\prime}}(m)=1$ and $\rho_{j-j^{\prime}}(b)=1$, respectively.

2.3.2. Example of Correlation Coefficient $\rho_{i-j, i^{\prime}-j^{\prime}}(\mathrm{bm})$. The $\rho_{i-j, i^{\prime}-j^{\prime}}(b m)$ in (10) contains the product of $\rho_{i-i^{\prime}}(m)$ and $\rho_{j-j^{\prime}}(b)$, which depend on the site environments and are independent of each other, though these environments might sometimes be the same. Therefore, to get $\rho_{i-j, i^{\prime}-j^{\prime}}(b m)$, it is sufficient to prepare just one side for various situations because we can get $\rho_{i-j, i^{\prime}-j^{\prime}}(\mathrm{bm})$ by combining them. Therefore, we studied the correlation for two typical distributions with uniform $\xi_{n}$ and Gaussian $\zeta_{n}$.

(i) For Uniform Distribution of $\xi_{n}$. We first calculate $\rho_{i-i^{\prime}}(m)$ by (9) when $\xi_{n}$ has a uniform distribution centered at $\xi_{c}$ over $\xi_{c}-\Delta \xi_{n}<\xi_{n}<\xi_{c}+\Delta \xi_{n}$ with the probability density function as $\operatorname{pdf}\left(\xi_{n}\right)=1 / 2 \Delta \xi_{n}$. Assuming a large $N$, we can calculate the ensemble average in (9) by integration with respect to $\xi_{n}$, and get (11) (see Appendix C).

$$
\begin{aligned}
\rho_{i-i^{\prime}}(m)=\sum_{l=0}^{\infty} \varepsilon_{l}(-1)^{l} J_{2 l}\left(2 \pi z_{i-i^{\prime}}\right) \frac{1}{2 l \Delta \xi_{n}} \sin \left(2 l \Delta \xi_{n}\right) \\
\quad \times \cos \left(2 l\left(\xi_{c}-\psi_{i-i^{\prime}}\right)\right) \\
-\quad j 2 \sum_{l=0}^{\infty}(-1)^{l} J_{2 l+1}\left(2 \pi z_{i-i^{\prime}}\right) \frac{1}{(2 l+1) \Delta \xi_{n}} \\
\quad \times \sin \left((2 l+1) \Delta \xi_{n}\right) \cos \left((2 l+1)\left(\xi_{c}-\psi_{i-i^{\prime}}\right)\right) .
\end{aligned}
$$

(ii) For Gaussian Distribution of $\zeta_{n}$. Next, we calculate $\rho_{j-j^{\prime}}(b)$ when $\zeta_{n}$ has a Gaussian distribution centered at $\zeta_{c}$ with deviation $\sigma$. Similar to the uniform distribution case, we get (12) (see Appendix D).

$$
\begin{aligned}
\rho_{j-j^{\prime}}(b)=\sum_{l=0}^{\infty} \varepsilon_{l}(-1)^{l} J_{2 l}\left(2 \pi z_{j-j^{\prime}}\right) \cos \left(2 l\left(\zeta_{c}-\psi_{j-j^{\prime}}\right)\right) \\
\quad \times \exp \left(-2 l^{2} \sigma^{2}\right) \\
-j 2 \sum_{l=0}^{\infty}(-1)^{l} J_{2 l+1}\left(2 \pi z_{j-j^{\prime}}\right) \\
\quad \times \cos \left((2 l+1)\left(\zeta_{c}-\psi_{j-j^{\prime}}\right)\right) \exp \left(-\frac{(2 l+1)^{2} \sigma^{2}}{2}\right) .
\end{aligned}
$$

\section{Simulation}

3.1. Simulation Method. A computer simulation was performed to verify (10), (11), and (12) and to study the relation between the MIMO channel matrix correlation and eigenvalue. The simulation parameters are listed in Table 1, assuming pico, micro, and macro cells indoors and outdoors. As suggested by (10), we need to simulate two items for the correlation: mobile or base station one-side channel, that is, $\rho_{i-i^{\prime}}(m)$ or $\rho_{j-j^{\prime}}(b)$, and mobile and base stations both-side channel, that is, $\rho_{i-j, i^{\prime}-j^{\prime}}(b m)$. So we simulated the correlation with uniform-in- $\xi_{n}$ and Gaussian-in- $\zeta_{n}$ distributions for each item. Concerning the eigenvalue, its dependence on the correlation was simulated while changing the mobile and base site conditions and environments. The radio frequency $f_{c}$ was $3 \mathrm{GHz}$, and the channel model with the delay profile in Section 2.1 was used. The nondirective wave amplitude $h_{n}$ exponentially decreases with increasing $\tau_{n}$, and the effective amplitude is greater than $-25 \mathrm{~dB}$ relative to the maximum one. The simulation was performed using (1), (2), (3), and (4); the incident and arriving angles $\left(\xi_{n}, \zeta_{n}\right)$ and antenna parameters $\left(\lambda_{m}, \xi_{m}, \lambda_{b}, \zeta_{b}\right)$ were set as shown in Table 1. Each simulated value was calculated from an ensemble average for more than $10^{6}$ delay profiles, except for eigenvalue variation with movement in Section 3.3. 


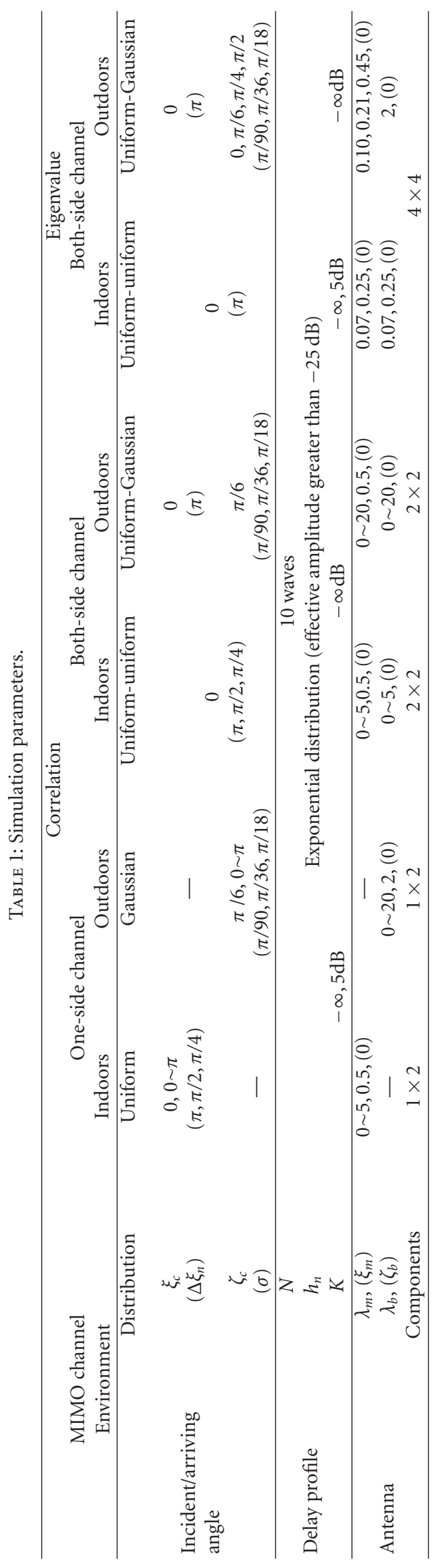




\subsection{Correlation Coefficient of MIMO Channel}

3.2.1. Mobile or Base Station One-Side Channel. Figure 3 shows the absolute value of the simulated correlation for a mobile- or base-station one-side channel assuming a mobile station indoors for Figures 3(a) and 3(b) and a base station outdoors for Figure 3(c), when the antenna's spacing $z=z_{i-i^{\prime}}\left(z_{j-j^{\prime}}\right)$ and setting angle $\Psi_{i-i^{\prime}}\left(\Psi_{j-j^{\prime}}\right)=0$. Figures 3(a) and 3(b) have arriving angle $\xi_{n}$ with uniform distribution and the parameter $\Delta \xi_{n}$ means the $n$th arriving wave uniformly from the direction $\xi_{c}-\Delta \xi_{n}<\xi_{n}<\xi_{c}+\Delta \xi_{n}$. Figures $3(\mathrm{a})$ and $3(\mathrm{~b})$ are for NLOS and LOS with $K=$ $5 \mathrm{~dB}$ and $\xi_{0}=5 \pi / 6$ paths, respectively, and the correlation of NLOS fluctuates less with increasing $z$, but that of LOS is higher than that of NLOS and is close to a fixed value $k /(k+1)$ fluctuating with increasing $z$. Figures 3(a) and 3 (b) also show the theoretical value $\left|\rho_{i-i^{\prime}}(m)\right|$ calculated by (10) with (11); the simulated and theoretical values agree well. The $\rho_{i-i^{\prime}}(m)$ at $\Delta \xi_{n}=\pi$ in Figure 3(a) becomes $J_{o}\left(2 \pi z_{i-i^{\prime}}\right)$, as is well known. Figure $3(\mathrm{c})$ shows the simulated correlation for arriving angle $\zeta_{n}$ with Gaussian distribution centered at $\zeta_{c}=\pi / 6$ with standard deviation $\sigma$. The correlation value decreases monotonically with increasing $z$ and the simulated values agree well with the theoretical values $\left|\rho_{j-j^{\prime}}(b)\right|$ obtained by (10) using(12).

Figure 4 shows the dependence of the correlation on centered arriving angle in NLOS for a one-side channel. Figures 4(a) and 4(b) are the cases for angle $\xi_{n}$ with uniform distribution centered on $\xi_{c}$ with $\Delta \xi_{n}$, and angle $\zeta_{n}$ with Gaussian distribution centered on $\zeta_{c}$ with $\sigma$, respectively. The correlation in Figure 4(a) was simulated by changing $\xi_{c}$ over the range from 0 to $\pi$ while keeping $z_{i-i^{\prime}}=0.5$ and $\Psi_{i-i^{\prime}}=0$. The correlation values for $\Delta \xi_{n}=\pi / 4$ and $\pi / 2$ have minima at $\xi_{c}=\pi / 2$ and become larger far away from $\xi_{c}=\pi / 2$, but the value for $\Delta \xi_{n}=\pi$ does not depend on $\xi_{c}$ since arriving waves arrive from all directions from 0 to $2 \pi$. The theoretical value $\left|\rho_{i-i^{\prime}}(m)\right|$ was calculated using (10), that is, (11); the theoretical and simulated values agree well. The correlation $\left|\rho_{j-j^{\prime}}(b)\right|$ in Figure 4(b) for the Gaussian distribution was also simulated in a similar way to Figure 4(a), expect for $z_{j-j^{\prime}}=2$. Though $\left|\rho_{j-j^{\prime}}(b)\right|$ has a minimum at $\xi_{c}=$ $\pi / 2$ and becomes larger far away from $\zeta_{c}=\pi / 2$ like in Figure $4(\mathrm{a})$, the values at $\zeta_{c}=\pi / 2$ depend on standard deviation $\sigma$, and the minimum value of $\left|\rho_{j-j^{\prime}}(b)\right|$ is large when $\sigma$ is small. The theoretical value was calculated using (10), that is, (12); the theoretical and simulated values agree well.

3.2.2. Mobile and Base Station Both-Side Channel. Figure 5 shows the simulated correlation coefficient $\left|\rho_{i-j, i^{\prime}-j^{\prime}}(b m)\right|$, that is, $i \neq i^{\prime}$ at the mobile station and $j \neq j^{\prime}$ at the base station for incident $\zeta_{n}$ and arriving $\xi_{n}$ with the same value of $\Delta \xi_{n}=\Delta \zeta_{n}$ in the both-side uniform distribution, assuming that the mobile and base stations are indoors. Figure 5(a) shows the simulated correlation when changing $z=z_{i-i^{\prime}}=$ $z_{j-j^{\prime}}$ and keeping $\Psi_{i-i^{\prime}}=\Psi_{j-j^{\prime}}=0$. The correlation decreases faster than in Figure 3(a). The reason for this can be seen from the theory that the theoretical value $\rho_{i-j, i^{\prime}-j^{\prime}}(b m)$

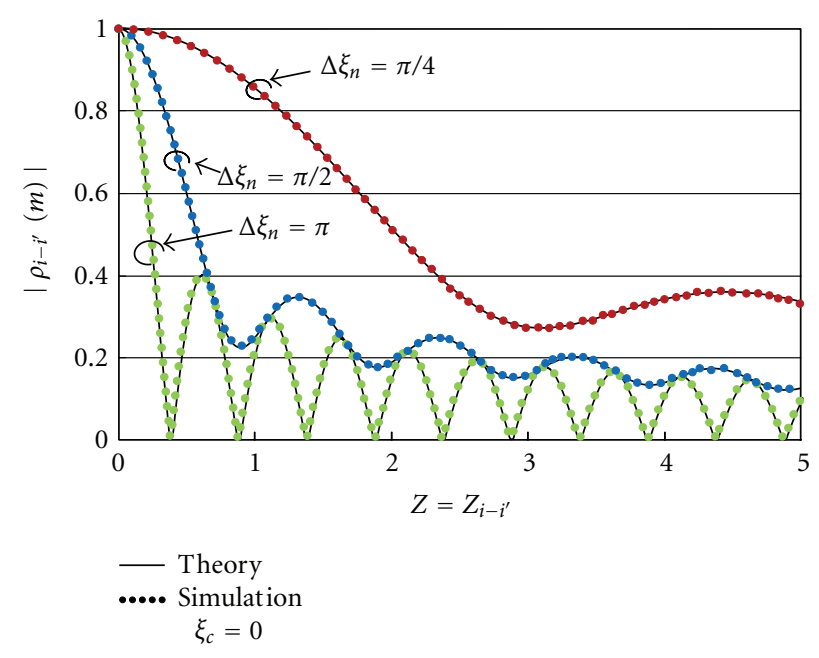

(a)

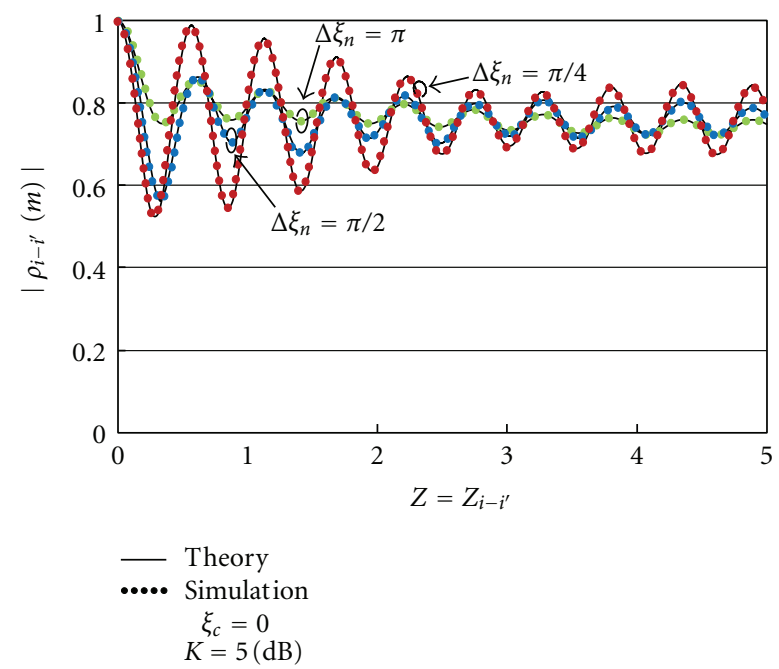

(b)

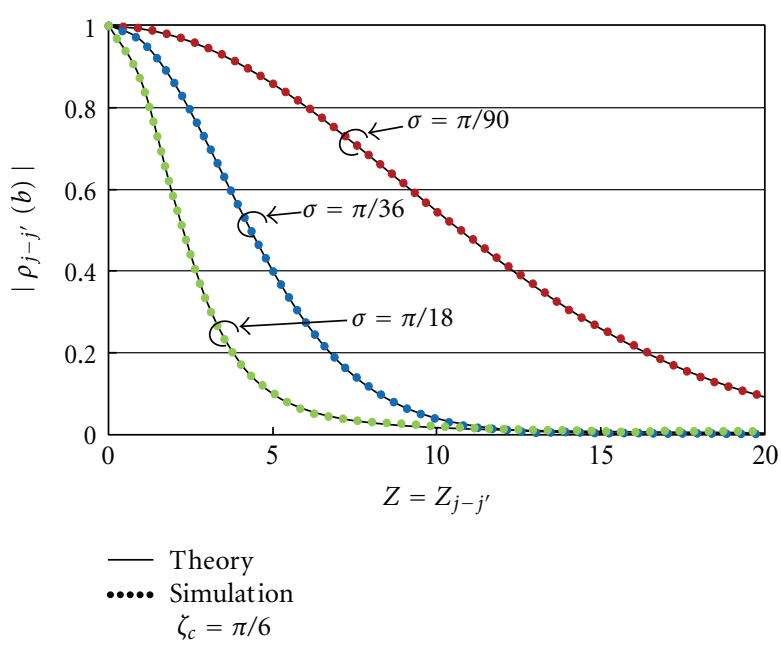

(c)

FIgure 3: Correlation of one-side channel. (a) NLOS indoors (uniform distribution). (b) LOS indoors (uniform distribution). (c) NLOS outdoors (Gaussian distribution). 


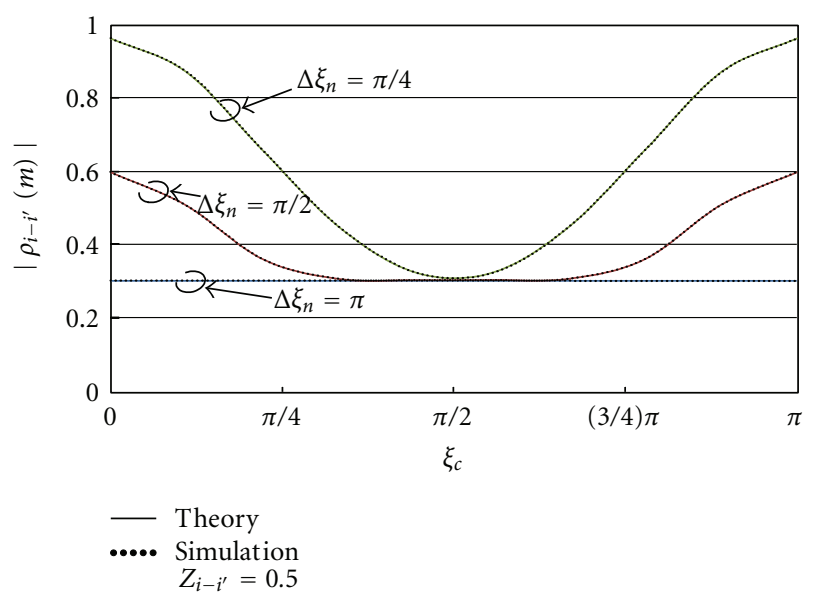

(a)

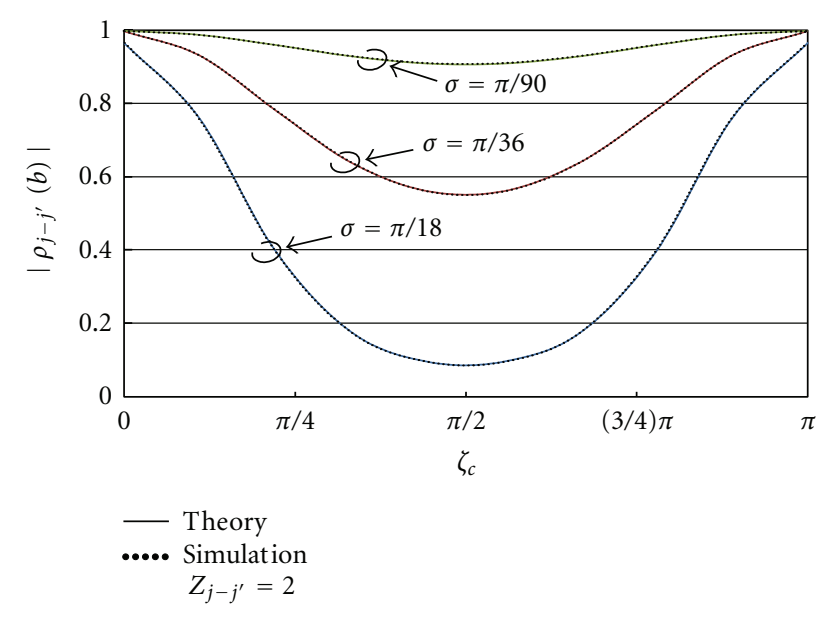

(b)

Figure 4: Correlation of dependence on arrival angle for one-side channel. (a) Uniform distribution. (b) Gaussian distribution.

from (10) is the product of $\rho_{i-i^{\prime}}(m)$ and $\rho_{j-j^{\prime}}(b)$, or here $\left|\rho_{i-j, i^{\prime}-j^{\prime}}(b m)\right|=\left|\rho_{i-i^{\prime}}(m)\right|^{2}$. The simulated and theoretical values agree well. Figure 5(b) is the simulated value when changing $z=z_{j-j^{\prime}}$, like Figure 5(a), except keeping $z_{i-i^{\prime}}=$ 0.5 . So the value of the correlation at $z=0$ is less than 1 . The theoretical values from (10) at $z_{i-i^{\prime}}=0.5$ are also shown in Figure 5(b); the theoretical and simulated values agree well.

Figure 6 shows the simulated correlation $\left|\rho_{i-j, i^{\prime}-j^{\prime}}(b m)\right|$ for incident $\xi_{n}$ with uniform distribution $\left(\xi_{c}=0, \Delta \xi_{n}=\right.$ $\pi)$ and arriving $\zeta_{n}$ with Gaussian distribution $\left(\zeta_{c}=\pi / 6\right.$, $\sigma$ : parameter), assuming mobile and base sites outdoors. Figure 6(a) was simulated like Figure 5(a), that is, changing $z$ and keeping $\sigma$ fixed; compared with those in Figure 3(c), the simulated values become small rapidly with increasing $z$ owing to the $\xi_{n}$ with uniform distribution at another site. The theoretical value $\rho_{i-j, i^{\prime}-j^{\prime}}(\mathrm{bm})$ from (10) was calculated as corresponding to (11) for the mobile station $\rho_{i-i^{\prime}}(m)$ and to (12) for the base station $\rho_{j-j^{\prime}}(b)$, respectively. The theoretical and simulated values agree well. Figure $6(\mathrm{~b})$ is the simulated correlation with changing $z=z_{j-j^{\prime}}$ as in Figure 6(a), except

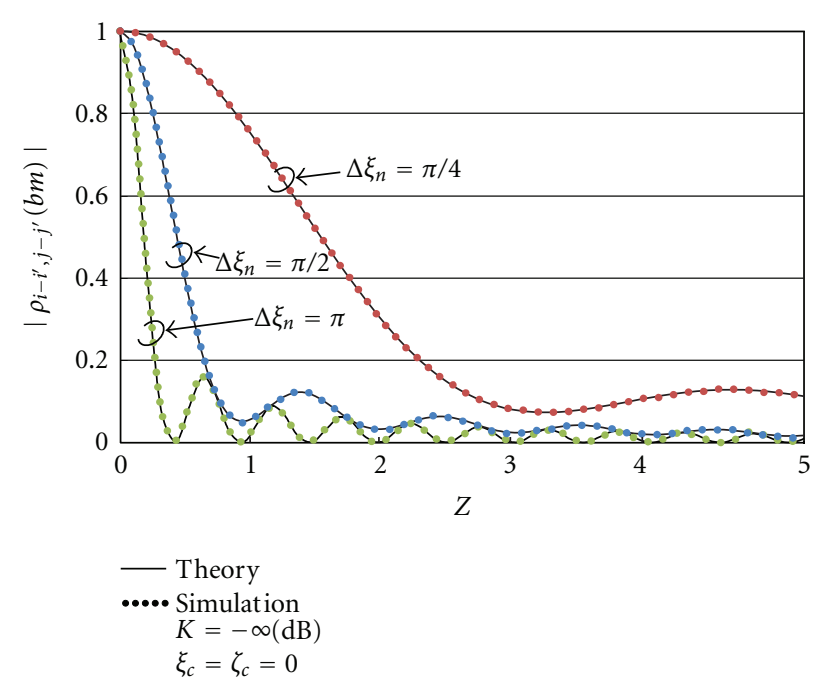

(a)
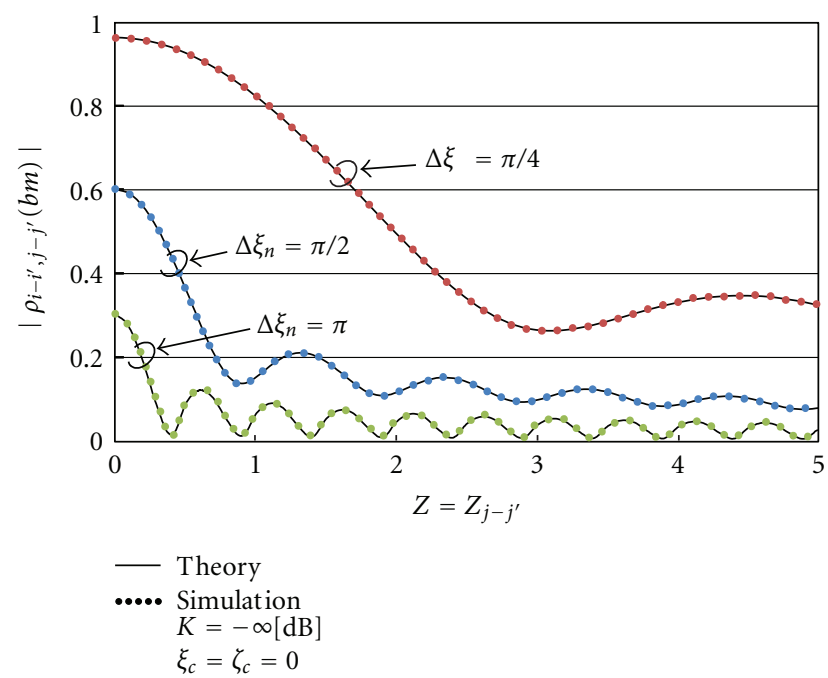

(b)

FIGURE 5: Correlation of both-side channel (uniform-uniform distribution). (a) $Z=Z_{i-i^{\prime}}=Z_{j-j^{\prime}}$. (b) $Z_{i-i^{\prime}}=0.5$.

keeping $z_{i-i^{\prime}}=0.5$. So the simulated value is less than 0.3 whenever $z$ is small since we kept $z_{i-i^{\prime}}=0.5$. The theoretical value from (10) was also calculated; the theoretical and simulated values agree well.

3.3. Eigenvalue of MIMO Channel Matrix. All of the eigenvalues were simulated by a MIMO antenna with a $4 \times$ 4 element configuration with elements placed with equal spacing and on a line in order to obtain the basic to eigenvalue properties.

3.3.1. Eigenvalue Property with Movement. Figure 7 shows an example of eigenvalue variation with movement of the mobile station calculated every 0.05 wavelength on the MIMO channel matrix by (3) in multipath fading. Figures $7(\mathrm{a})$ and $7(\mathrm{~b})$ were simulated using the same delay profile 


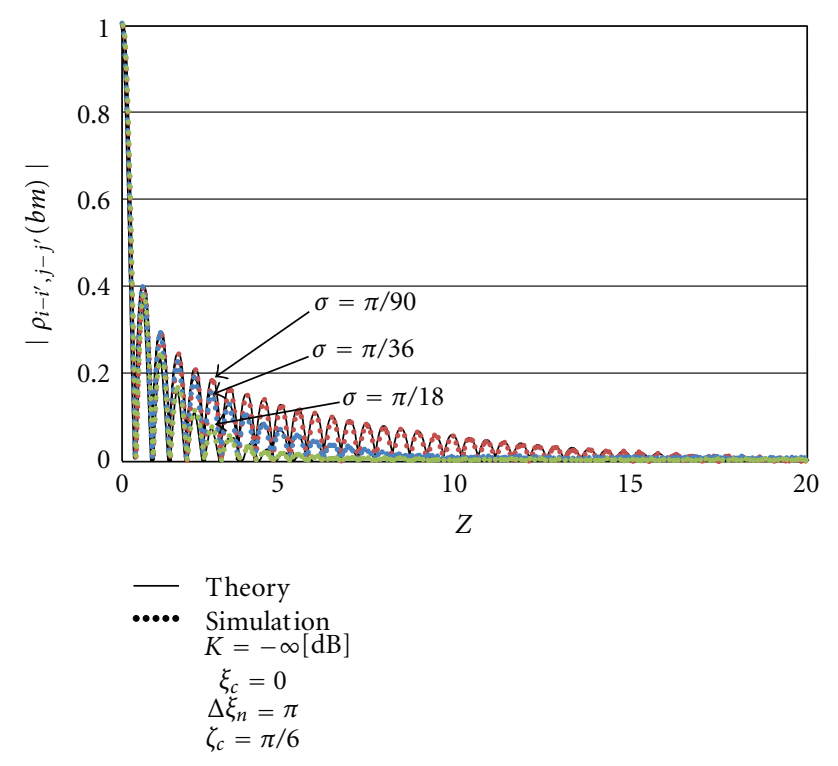

(a)

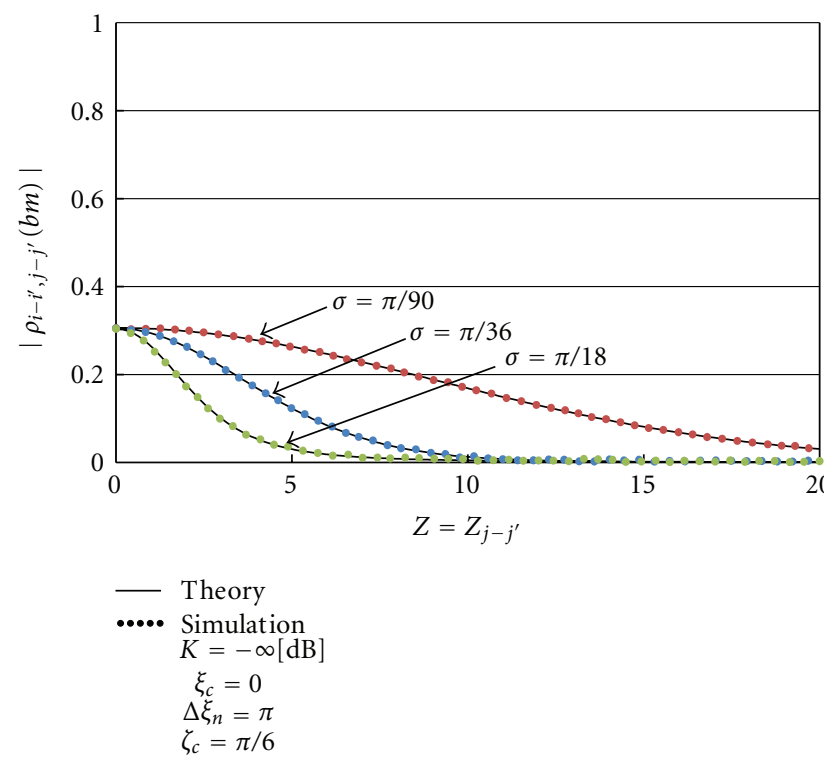

(b)

Figure 6: Correlation of both-side channel (uniform-Gaussian distribution). (a) $Z=Z_{i-i^{\prime}}=Z_{j-j^{\prime}}$. (b) $Z_{i-i^{\prime}}=0.5$.

with both-side uniform distribution with $\xi_{c}=0$ and $\Delta \xi_{n}=\pi$ in NLOS; the only difference was the antenna radius $\lambda_{m i}=\lambda_{b j}=0.25$ and 0.07 to make low and high correlations by (11) or the theoretical correlation between one MIMO antenna element and the next one $\left|\rho_{i-i+1}(m)\right|=$ $\left|\rho_{j-j+1}(b)\right|=0.45$ and 0.95 , respectively. Here, $\rho$ in Figure 7 means $\left|\rho_{i-j, i^{\prime}-j^{\prime}}(\mathrm{bm})\right|$ from (10). Moreover, Figure 7(c) was simulated under the condition in Figure $7(\mathrm{a})$ with just the addition of a direct wave with $K=5[\mathrm{~dB}]$ to the delay profile, when the total power of the profile was normalized to 1 , and the $\rho$ by (10) is 0.7. Comparing Figures 7(a) and 7(b), we see that the first eigenvalue $\lambda_{1}$ at $\rho=0.2$ in Figure 7(a) is almost

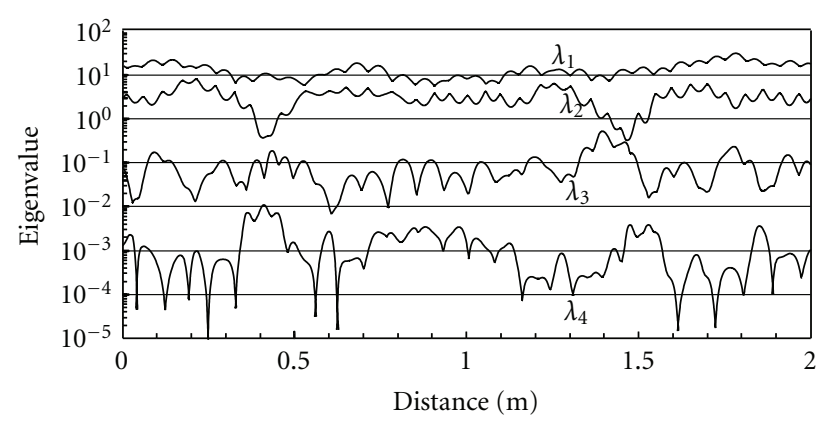

(a)

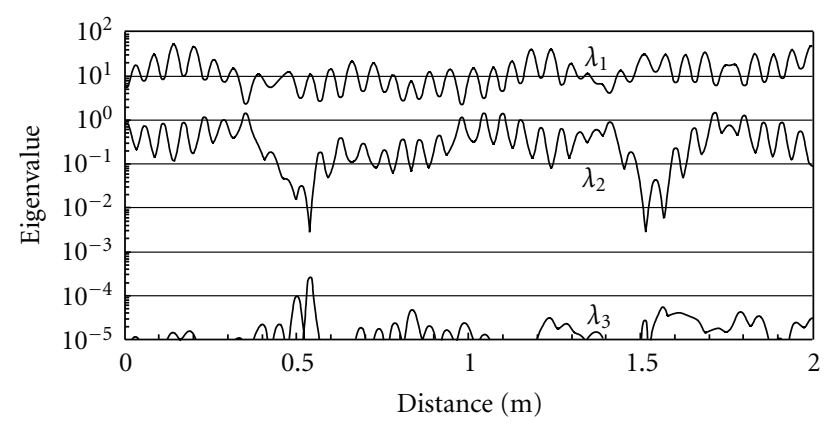

(b)

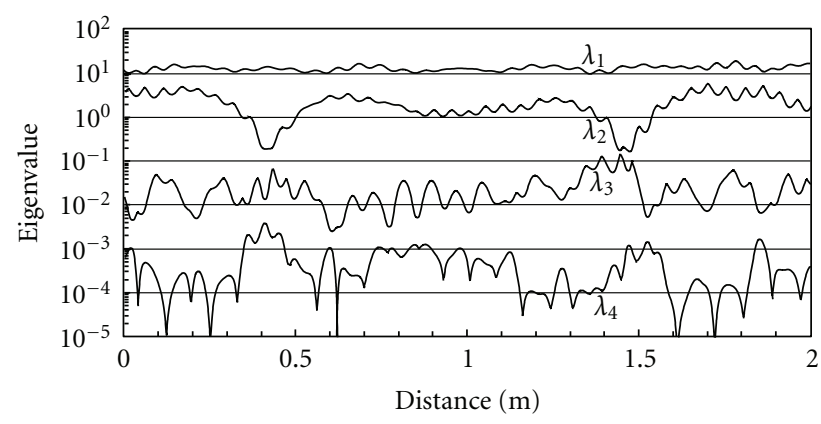

(c)

FIGURE 7: Example of eigenvalue variation with movement $(4 \times 4$ MIMO, uniform distribution). (a) NLOS $(\rho=0.2)$. (b) NLOS ( $\rho=$ $0.9)$. (c) $\operatorname{LOS}(K=5[\mathrm{~dB}], \rho=0.7)$.

equal to that at $\rho=0.9$ in Figure 7(b) on average but that the other eigenvalues $\lambda_{2}, \lambda_{3}$, and $\lambda_{4}$ in Figure $7(\mathrm{a})$ are larger than that in Figure $7(\mathrm{~b})\left(\lambda_{4}\right.$ is less than $\left.10^{-5}\right)$. Moreover, each eigenvalue variation with movement in Figure 7(a) is smaller than the corresponding one in Figure 7(b). On the other hand, the average value of each eigenvalue in Figure $7(\mathrm{c})$ in LOS seems to be similar to that in Figure 7(a), but the state is more stable, especially for $\lambda_{1}$, than that in NLOS.

3.3.2. Dependence of Eigenvalue on Correlation. Figure 8 shows the dependence of the eigenvalue on correlation with the property of antenna space and multipath channel by a cumulative distribution. All the eigenvalue curves in Figure 8(a), that is, at $\rho=0.2$ and 0.9 in NLOS and at $\rho=0.7$ in LOS, were simulated under the same conditions as in Figures $7(\mathrm{a}), 7(\mathrm{~b})$, and $7(\mathrm{c})$, respectively, except for the use of only one delay profile, or at this time the use of 

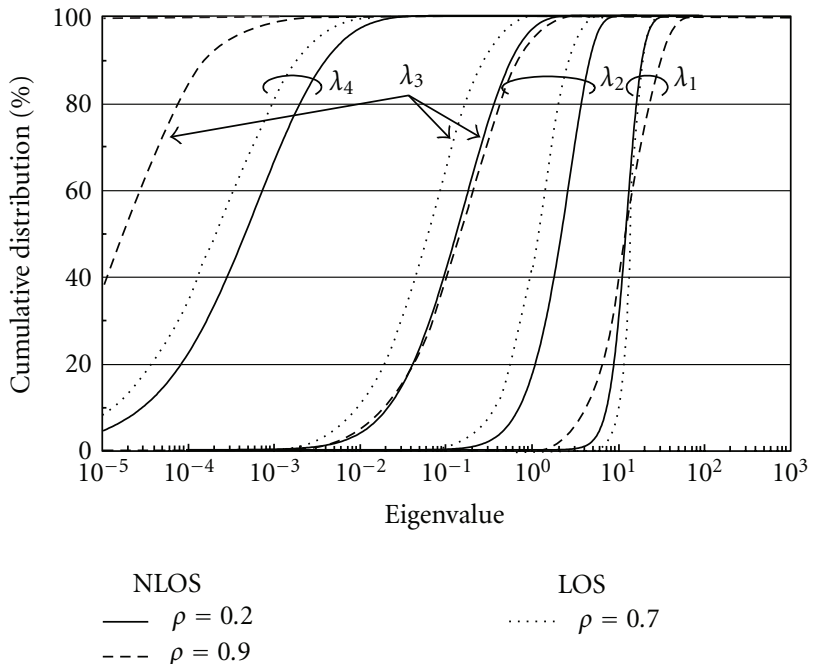

(a)
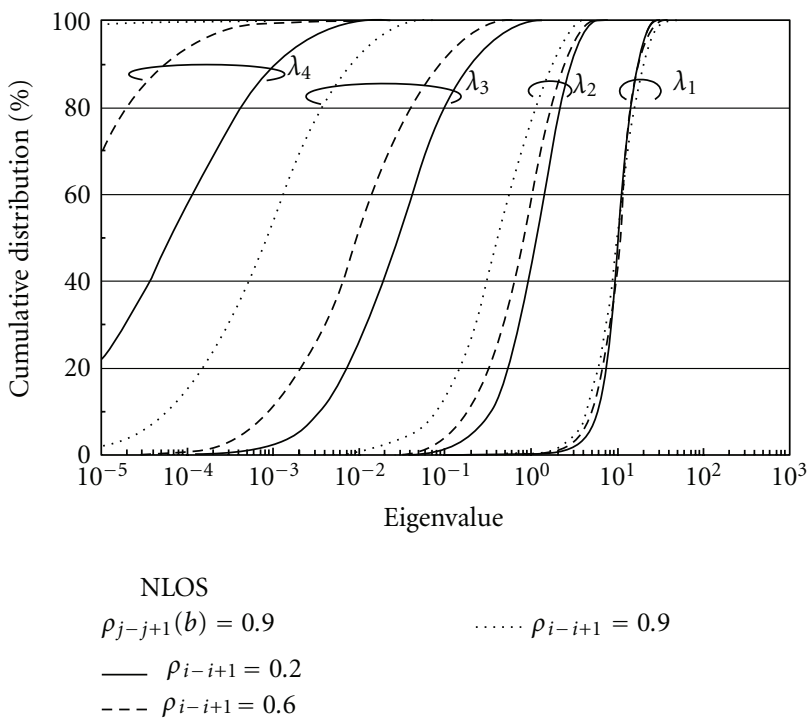

(b)

Figure 8: Dependence of eigenvalue on correlation $(4 \times 4$ MIMO). (a) Uniform-uniform distribution. (b) Uniform-Gaussian distribution.

more than $10^{6}$ profiles. As assumed in Figure 7, Figure 8(a) seems to suggest the following: though the $50 \%$ cumulative values in $\lambda_{1}$ are weakly dependent on $\rho$ and almost equal, the other eigenvalues $\lambda_{2}, \lambda_{3}$, and $\lambda_{4}$ are dependent on $\rho$ and $K$, and the $\lambda_{1}$ in LOS is the most stable. Figure $8(\mathrm{~b})$ shows the dependence of the eigenvalue on correlation for $\rho_{i-j, i^{\prime}-j^{\prime}}(b m)=\rho_{i-i+1}(m) \rho_{j-j+1}(b)$ in NLOS, where the simulation was done changing antenna space correspondent to $\left|\rho_{i-i+1}(m)\right|=0.2,0.6$, and 0.9 at the mobile station with a uniform distribution keeping a high $\left|\rho_{j-j+1}(b)\right|=0.9$ at the base station in the Gaussian distribution with $\zeta_{c}=\pi / 6$ and $\sigma=\pi / 36$. Figure $8(\mathrm{~b})$ also shows that the $50 \%$ cumulative values have little dependence on $\rho_{i-j, i^{\prime}-j^{\prime}}(\mathrm{bm})$ for $\lambda_{1}$, but are dependent on $\rho_{i-j, i^{\prime}-j^{\prime}}(\mathrm{bm})$ for the other eigenvalues: the

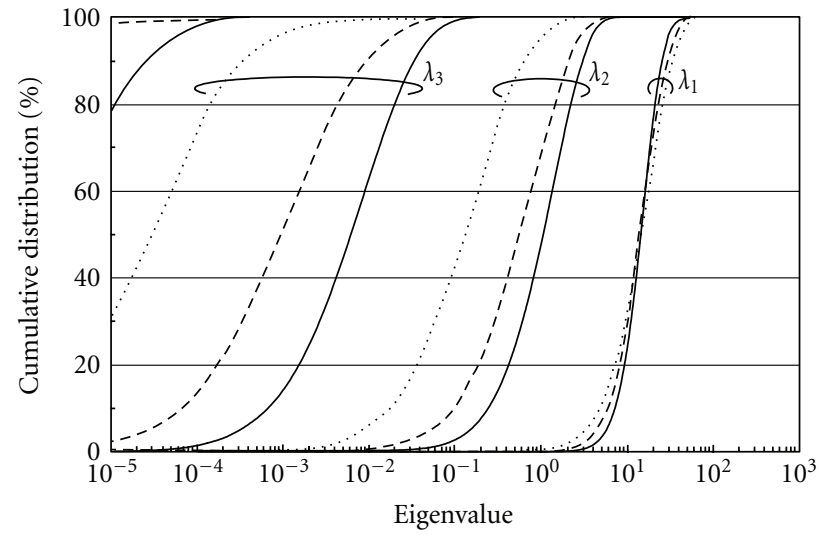

$$
\begin{gathered}
\text { NLOS } \\
\rho_{i-i+1}(m)=0.9 \\
Z_{j-j^{\prime}}=2 \\
-\sigma=\pi / 18 \\
---\sigma=\pi / 36 \\
\cdots \cdot \sigma=\pi / 90
\end{gathered}
$$

(a)

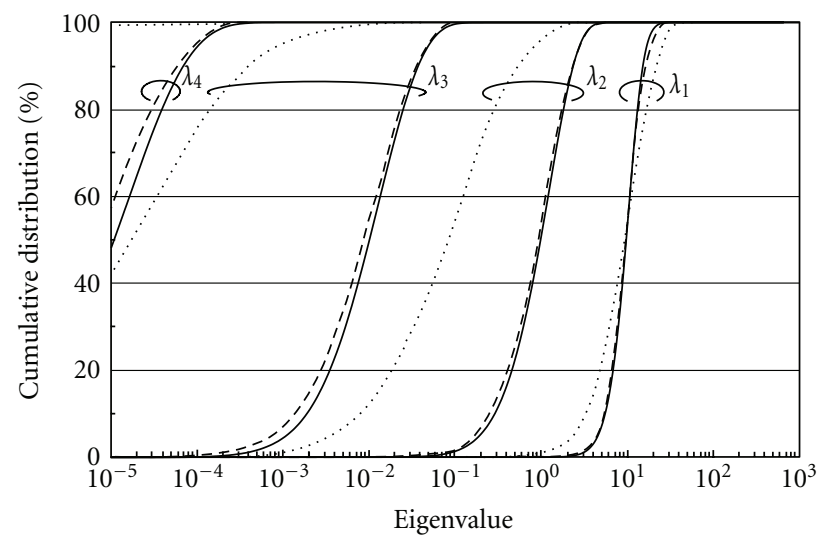

$$
\begin{gathered}
\text { NLOS } \\
\rho_{i-i+1}(m)=0.9 \\
Z_{j-j j^{\prime}}=2 \\
-\zeta_{c}=\pi / 2 \\
---\zeta_{c}=\pi / 4 \\
\ldots \ldots \zeta_{c}=0
\end{gathered}
$$

(b)

Figure 9: Dependence of eigenvalue on Gaussian distribution $(4 \times$ 4 MIMO). (a) Effect of standard deviation. (b) Effect of centering arriving angle.

smaller $\rho_{i-j, i^{\prime}-j^{\prime}}(b m)$ is, the closer the values are to $\lambda_{1}$. All the eigenvalues tend to have a wider distribution with increasing $\rho_{i-j, i^{\prime}-j^{\prime}}(b m)$.

Figure 9 shows the dependence of the eigenvalue on correlation $\rho_{j-j+1}(b)$ with the properties of standard deviation $\sigma$ and centering angle $\zeta_{c}$ in Gaussian distribution, as shown in Figure 4(b). Simulation was done for $\rho_{j-j+1}(b)$ with $\sigma$ and $\zeta_{c}$ as parameters, while the mobile station condition was set to a constant at $\left|\rho_{i-i+1}(m)\right|=0.9$ in a uniform distribution with $\xi_{c}=0$ and $\Delta \xi_{n}=\pi$. Figure 9(a) shows the influence 
of $\sigma$ on the eigenvalue with $\sigma=\pi / 18, \pi / 36$, and $\pi / 90$ as parameters at $\zeta_{c}=\pi / 6$ and $z_{j-j^{\prime}}=2$. These $50 \%$ cumulative values are also weakly dependent on $\sigma$ for $\lambda_{1}$, but do depend on $\sigma$ for the other eigenvalues, and they become larger when $\sigma$ becomes large, that is, $\rho_{j-j+1}(b)$ is small. Figure 9 (b) shows the influence of $\zeta_{c}$ on the eigenvalue, when $\zeta_{c}=\pi / 2, \pi / 4$, and 0 at $\sigma=\pi / 18$ and $z_{j-j^{\prime}}=2$. The dependence of the eigenvalues on $\zeta_{c}$ is similar to $\sigma$ in Figure 9(a). Figure 9 suggests that the $\sigma$ and $\zeta_{c}$ are parameters for eigenvalue property.

\section{Conclusion}

To study MIMO channel properties, we proposed a MIMO channel model with a propagating mechanism composed of multipath propagation and antenna configurations and then showed the MIMO channel matrix. Under this model, the spatial correlation formula $\rho_{i-j, i^{\prime}-j^{\prime}}(\mathrm{bm})$ between MIMO channel matrix elements was derived: the formula $\rho_{i-j, i^{\prime}-j^{\prime}}(\mathrm{bm})$ was expressed as a directive wave term added to the product of mobile site correlation $\rho_{i-i^{\prime}}(m)$ and base site correlation $\rho_{j-j^{\prime}}(b)$, which are calculated independently of each other, divided by $k+1$. This formula can be applied to create the channel matrix element with a fixed value of correlation estimated by $\rho_{i-j, i^{\prime}-j^{\prime}}(\mathrm{bm})$ for given multipath conditions and antenna configurations. Furthermore, simulation was done for the correlation and channel matrix eigenvalue indoors, outdoors, and for movement. The simulated and theoretical values of the correlation agree well. The simulated eigenvalue shows that the average of the first eigenvalue $\lambda_{1}$ is hardly dependent on the correlation $\rho_{i-j, i^{\prime}-j^{\prime}}(\mathrm{bm})$, but the other ones are dependent on $\rho_{i-j, i^{\prime}-j^{\prime}}(\mathrm{bm})$ and become close to $\lambda_{1}$ with decreasing $\rho_{i-j, i^{\prime}-j^{\prime}}(b m)$. Moreover, the $\lambda_{1}$ state with mobile movement in LOS path is more stable than the $\lambda_{1}$ of NLOS path. The MIMO channel model and derived $\rho_{i-j, i^{\prime}-j^{\prime}}(\mathrm{bm})$ make it possible to create a MIMO channel matrix with a fixed value correlation and furthermore to study the relation between the correlation and eigenvalue for various cell sites and environments.

\section{Appendices}

\section{A. Derivation of (5)}

Assuming that $N$ is a large number for the delay profile in Section 2.1, then because $\tau_{n}$ and $\phi_{n}$ have random values, $\theta_{i-j, n}$ obtained by (2) is a random value over the range from 0 to $2 \pi$ and independent of $h_{n}$. So we get $\langle x\rangle=\left\langle\sum_{n=0}^{N} h_{n}\right\rangle\left\langle\sum_{n=0}^{N} \exp \left(j \theta_{i-j, n}\right)\right\rangle=0$ since the term $\left\langle\sum_{n=0}^{N} \exp \left(j \theta_{i-j, n}\right)\right\rangle$ becomes zero, that is, $\langle x\rangle$ and $\langle y\rangle$ in (4) are zero. Therefore, the denominator in (4) becomes $\left[\left\langle x^{*} x\right\rangle\left\langle y^{*} y\right\rangle\right]^{1 / 2}$, that is, $k+1$. On the other hand, $\left\langle(x-\langle x\rangle)^{*}(y-\langle y\rangle)\right\rangle$ in the numerator in (4) can be modified to $\left\langle x^{*} y\right\rangle=\left\langle E_{i-j}\left(t, f_{c}\right)^{*} E_{i^{\prime}-j^{\prime}}\left(t, f_{c}\right)\right\rangle$. As a result, the sum of products $E_{i-j}\left(t, f_{c}\right)^{*}$ and $E_{i^{\prime}-j^{\prime}}\left(t, f_{c}\right)$ remains the same for the $n$th arriving wave on MIMO $\operatorname{ch}(i-j)$ and $\operatorname{ch}\left(i^{\prime}-j^{\prime}\right)$, but vanishes each other differences for the $n$th arriving wave on MIMO $\operatorname{ch}(i-j)$ and $\operatorname{ch}\left(i^{\prime}-j^{\prime}\right)$ because the values of $\theta_{i^{\prime}-j^{\prime}, n^{\prime}}-\theta_{i-j, n}$ are random; moreover, $h_{n} h_{n^{\prime}}$ and $\theta_{i^{\prime}-j^{\prime}, n^{\prime}}-\theta_{i-j, n}$ are independent of each other. Therefore, we get (A.1) while considering $h_{n}$ and $\Delta \theta_{n}$; moreover, mobile and base site factors are independent of each other, and the directive wave is deterministic and the amplitude $h_{0}$ is much larger than the others $h_{n}$.

$$
\begin{aligned}
\langle & \left.(x-\langle x\rangle)^{*}(y-\langle y\rangle)\right\rangle \\
= & \left\langle E_{i-j}\left(t, f_{c}\right)^{*} E_{i^{\prime}-j^{\prime}}\left(t, f_{c}\right)\right\rangle \\
= & \left\langle\sum_{n=0}^{N} h_{n}^{2} \exp \left(j \Delta \theta_{n}\right)+\sum_{n=0}^{N^{\prime}} \sum_{n^{\prime}=0}^{N^{\prime}} h_{n} h_{n^{\prime}}\right. \\
& \left.\times \exp \left(j\left(\theta_{i^{\prime}-j^{\prime}, n^{\prime}}-\theta_{i-j, n}\right)\right)\right\rangle \\
= & \left\langle\sum_{n=0}^{N} h_{n}^{2} \exp \left(j \Delta \theta_{n}\right)\right\rangle \\
= & \left\langle h_{0}^{2} \exp \left(j \Delta \theta_{0}\right)\right\rangle \\
& +\left\langle\sum_{n=1}^{N} h_{n}^{2}\right\rangle\left\langle\sum _ { n = 1 } ^ { N } \operatorname { e x p } \left[-j 2 \pi\left\{\lambda_{m i^{\prime}} \cos \left(\xi_{n}-\xi_{m i^{\prime}}\right)\right.\right.\right. \\
& \times\left\langle\sum _ { n = 1 } ^ { N } \operatorname { e x p } \left[-j 2 \pi\left\{\lambda_{b j^{\prime}} \cos \left(\zeta_{n}-\zeta_{b j^{\prime}}\right)\right.\right.\right. \\
\left.\left.\left.-\lambda_{m i} \cos \left(\xi_{n}-\xi_{m i}\right)\right\}\right]\right\rangle & \left.\left.\left.\cos \left(\zeta_{n}-\zeta_{b j}\right)\right\}\right]\right\rangle \\
&
\end{aligned}
$$

where $n \neq n^{\prime}$ in $\sum_{n=0}^{N}{ }^{\prime} \sum_{n^{\prime}=0}^{N}$.

$$
\begin{aligned}
\Delta \theta_{n}=-2 \pi[ & \lambda_{m i^{\prime}} \cos \left(\xi_{n}-\xi_{m i^{\prime}}\right)+\lambda_{b j^{\prime}} \cos \left(\zeta_{n}-\zeta_{b j^{\prime}}\right) \\
& \left.-\lambda_{m i} \cos \left(\xi_{n}-\xi_{m i}\right)-\lambda_{b j} \cos \left(\zeta_{n}-\zeta_{b j}\right)\right] .
\end{aligned}
$$

The $\Delta \theta_{n}$ yielded by (A.2) expresses the phase difference between MIMO $\operatorname{ch}\left(i^{\prime}-j^{\prime}\right)$ and $\operatorname{ch}(i-j)$ for the $n$th arriving wave, or $\Delta \theta_{n}=\theta_{i^{\prime}-j^{\prime}, n}-\theta_{i-j, n}$. Equation (A.1) has two terms, that is, directive and no directive wave terms. Moreover, since the second term can be classified into no directive power, mobile-site, and base-site factors, the classified factors are calculated independently of each other. So we used the ensemble average of mobile and base sites as $\rho_{i-i^{\prime}}(m)$ and $\rho_{j-j^{\prime}}(b)$, respectively, where $\left\langle\sum_{n=1}^{N} h_{n}^{2}\right\rangle=1$.

Furthermore, by continuously analyzing $\rho_{i-i^{\prime}}(m)$, expanding $\cos \left(\xi_{n}-\xi_{m i^{\prime}}\right)$ and $\cos \left(\xi_{n}-\xi_{m i}\right)$ in (A.1), and gathering terms of $\cos \xi_{n}$ and $\sin \xi_{n}$, and with a little modification we get (A.3) and express that as the third 
ensemble average in (5).

$$
\begin{aligned}
\rho_{i-i^{\prime}}(m)=\left\langle\sum _ { n = 1 } ^ { N } \operatorname { e x p } \left[-j 2 \pi\left\{\lambda_{m i^{\prime}} \cos \left(\xi_{n}-\xi_{m i^{\prime}}\right)\right.\right.\right. \\
\left.\left.\left.-\lambda_{m i} \cos \left(\xi_{n}-\xi_{m i}\right)\right\}\right]\right\rangle \\
=\left\langle\sum _ { n = 1 } ^ { N } \operatorname { e x p } \left[-j 2 \pi\left\{\left(\lambda_{m i^{\prime}} \cos \xi_{m i^{\prime}}-\lambda_{m i} \cos \xi_{m i}\right) \cos \xi_{n}\right.\right.\right. \\
\left.\left.\left.+\left(\lambda_{m i^{\prime}} \sin \xi_{m i^{\prime}}-\lambda_{m i} \sin \xi_{m i}\right) \sin \xi_{n}\right\}\right]\right\rangle \\
=\left\langle\sum_{n=1}^{N} \exp \left[-j 2 \pi z_{i-i^{\prime}} \cos \left(\xi_{n}-\psi_{i-i^{\prime}}\right)\right]\right\rangle .
\end{aligned}
$$

Here, $z_{i-i^{\prime}}$ and $\Psi_{i-i^{\prime}}$ are antenna construction parameters obtained from (7) for a mobile site. The $\rho_{j-j^{\prime}}(b)$ for the base site is calculated in a similar manner to $\rho_{i-i^{\prime}}(m)$.

\section{B. Derivation of $(9)$}

We expand the real and imaginary parts for $\rho_{i-i^{\prime}}(m)$ in (5) into a Neumann expansion and get (9).

$$
\begin{aligned}
\rho_{i-i^{\prime}}(m)=\left\langle\sum _ { n = 1 } ^ { N } \left[\cos \left\{2 \pi z_{i-i^{\prime}} \cos \left(\xi_{n}-\psi_{i-i^{\prime}}\right)\right\}\right.\right. \\
\left.\left.\quad-j \sin \left\{2 \pi z_{i-i^{\prime}} \cos \left(\xi_{n}-\psi_{i-i^{\prime}}\right)\right\}\right]\right\rangle \\
=\left\langle\sum_{n=1}^{N}\left[\sum_{l=0}^{\infty} \varepsilon_{l}(-1)^{l} J_{2 l}\left(2 \pi z_{i-i^{\prime}}\right) \cos \left(2 l\left(\xi_{n}-\psi_{i-i^{\prime}}\right)\right)\right]\right\rangle \\
\quad-j\left\langle\sum_{n=1}^{N}\left[2 \sum_{l=0}^{\infty}(-1)^{l} J_{2 l+1}\left(2 \pi z_{i-i^{\prime}}\right)\right]\right\rangle \\
\left.\left.\times \cos \left((2 l+1)\left(\xi_{n}-\psi_{i-i^{\prime}}\right)\right)\right]\right\rangle .
\end{aligned}
$$

\section{Derivation of (11)}

The real and imaginary parts of $\rho_{i-i^{\prime}}(m)$ in (B.1) are denoted $A$ and $B$. First, we calculate the real part $A$. Assuming a large $N$, when $\xi_{n}$ has a uniform distribution centered at $\xi_{c}$ over $\xi_{c}-$ $\Delta \xi_{n}<\xi_{n}<\xi_{c}+\Delta \xi_{n}$, and the probability density function of $\xi_{n}$ is $p d f\left(\xi_{n}\right)=1 / 2 \Delta \xi_{n}$, we can calculate the ensemble average in (9) by integration with respect to $\xi_{n}$, replacing variable $\xi_{n}-$ $\Psi_{i-i^{\prime}}$ by $u$.

$$
\begin{aligned}
\rho_{i-i^{\prime}}(m)= & A-j B \\
A= & \left\langle\sum_{n=1}^{N}\left[\sum_{l=0}^{\infty} \varepsilon_{l}(-1)^{l} J_{2 l}\left(2 \pi z_{i-i^{\prime}}\right) \cos \left(2 l\left(\xi_{n}-\psi_{i-i^{\prime}}\right)\right)\right]\right\rangle \\
= & \sum_{l=0}^{\infty} \varepsilon_{l}(-1)^{l} J_{2 l}\left(2 \pi z_{i-i^{\prime}}\right) \frac{1}{2 \Delta \xi_{n}} \\
& \times \int_{\xi_{c}-\Delta \xi_{n}}^{\xi_{c}+\Delta \xi_{n}} \cos \left(2 l\left(\xi_{n}-\psi_{i-i^{\prime}}\right)\right) d \xi_{n} \\
= & \sum_{l=0}^{\infty} \varepsilon_{l}(-1)^{l} J_{2 l}\left(2 \pi z_{i-i^{\prime}}\right) \frac{1}{2 \Delta \xi_{n}}\left[\frac{\sin (2 l u)}{2 l}\right]_{\xi_{c}-\psi_{i-i^{\prime}}-\Delta \xi_{n}}^{\xi_{c}-\psi_{i-i^{\prime}}+\Delta \xi_{n}} \\
= & \sum_{l=0}^{\infty} \varepsilon_{l}(-1)^{l} J_{2 l}\left(2 \pi z_{i-i^{\prime}}\right) \frac{1}{2 l \Delta \xi_{n}} \sin \left(2 l \Delta \xi_{n}\right) \\
& \times \cos \left(2 l\left(\xi_{c}-\psi_{i-i^{\prime}}\right)\right) .
\end{aligned}
$$

The imaginary part $B$ can be also calculated similarly to $A$; we get (C.2):

$$
\begin{aligned}
B=2 \sum_{l=0}^{\infty} & (-1)^{l} J_{2 l+1}\left(2 \pi z_{i-i^{\prime}}\right) \frac{1}{(2 l+1) \Delta \xi_{n}} \sin \left((2 l+1) \Delta \xi_{n}\right) \\
& \times \cos \left((2 l+1)\left(\xi_{c}-\psi_{i-i^{\prime}}\right)\right) .
\end{aligned}
$$

\section{Derivation of (12)}

The real and imaginary parts for $\rho_{j-j^{\prime}}(b)$ in (5) are denoted $A$ and $B$. We begin by calculating the real part $A$. Assuming that $\zeta_{n}$ has a Gaussian distribution centered at $\zeta_{c}$ with standard deviation $\sigma$ by (D.1), first the real part $A$ is expanded into a Neumann expansion and then the ensemble average is calculated by integration with respect to $\xi_{n}$ as follows.

$$
\begin{aligned}
& p d f: p\left(\zeta_{n}\right)=\frac{1}{\sqrt{2 \pi \sigma^{2}}} \exp \left(\frac{\left(\zeta_{n}-\zeta_{c}\right)^{2}}{2 \sigma^{2}}\right), \\
& \rho_{j-j^{\prime}}(b)=\left\langle\sum_{n=1}^{N} \exp \left[-j 2 \pi z_{j-j^{\prime}} \cos \left(\zeta_{n}-\psi_{j-j^{\prime}}\right)\right]\right\rangle \\
& =A-j B \text {, } \\
& A=\left\langle\sum_{n=1}^{N} \cos \left(2 \pi z_{j-j^{\prime}} \cos \left(\zeta_{n}-\psi_{j-j^{\prime}}\right)\right)\right\rangle \\
& =\left\langle\sum_{n=1}^{N}\left[\sum_{l=0}^{\infty} \varepsilon_{l}(-1)^{l} J_{2 l}\left(2 \pi z_{j-j^{\prime}}\right) \cos \left(2 l\left(\zeta_{n}-\psi_{j-j^{\prime}}\right)\right)\right]\right\rangle \\
& =\sum_{l=0}^{\infty} \varepsilon_{l}(-1)^{l} J_{2 l}\left(2 \pi z_{j-j^{\prime}}\right) \int_{\zeta_{c}-\pi}^{\zeta_{c}+\pi} \cos \left(2 l\left(\zeta_{n}-\psi_{j-j^{\prime}}\right)\right) \\
& \times p\left(\zeta_{n}\right) d \zeta_{n} .
\end{aligned}
$$


Putting $\zeta_{n}-\zeta_{c}=u$, with an odd function of sine and assuming $(\pi / 2 \sigma)^{2} \gg 1$ and with a little modification, we get

$$
\begin{aligned}
A= & \frac{1}{\sqrt{2 \pi \sigma^{2}}} \sum_{l=0}^{\infty} \varepsilon_{l}(-1)^{l} J_{2 l}\left(2 \pi z_{j-j^{\prime}}\right) \\
& \times \int_{-\pi}^{\pi} \cos \left(2 l\left(u+\zeta_{c}-\psi_{j-j^{\prime}}\right)\right) \exp \left(-\frac{u^{2}}{2 \sigma^{2}}\right) d u \\
= & \frac{1}{\sqrt{2 \pi \sigma^{2}}} \sum_{l=0}^{\infty} \varepsilon_{l}(-1)^{l} J_{2 l}\left(2 \pi z_{j-j^{\prime}}\right) \cos \left(2 l\left(\zeta_{c}-\psi_{j-j^{\prime}}\right)\right) \\
& \times \int_{-\pi}^{\pi} \cos (2 l u) \exp \left(-\frac{u^{2}}{2 \sigma^{2}}\right) d u \\
= & \sum_{l=0}^{\infty} \varepsilon_{l}(-1)^{l} J_{2 l}\left(2 \pi z_{j-j^{\prime}}\right) \cos \left(2 l\left(\zeta_{c}-\psi_{j-j^{\prime}}\right)\right) \\
& \times \exp \left(-2 l^{2} \sigma^{2}\right) .
\end{aligned}
$$

The imaginary part $B$ can also be calculated in a similar manner to $A$. We get (D.4).

$$
\begin{aligned}
B= & 2 \sum_{l=0}^{\infty}(-1)^{l} J_{2 l+1}\left(2 \pi z_{j-j^{\prime}}\right) \cos \left((2 l+1)\left(\zeta_{c}-\psi_{j-j^{\prime}}\right)\right) \\
& \times \exp \left(-\frac{(2 l+1)^{2} \sigma^{2}}{2}\right) .
\end{aligned}
$$

\section{References}

[1] ITU Circular Letter 5/LCCE/2, "Radiocommunication Bureau," March 2008.

[2] G. J. Foschini and M. J. Gans, "On limits of wireless communications in fading environments when using multiple antennas," Wireless Personal Communications, vol. 6, no. 3, pp. 311335, 1998.

[3] D. S. Shiu, G. J. Foschini, M. J. Gans, and J. M. Kahn, "Fading correlation and its effect on the capacity of multielement antenna systems," IEEE Transactions on Communications, vol. 48, no. 3, pp. 502-513, 2000.

[4] R. Nee and R. Prasad, OFDM for Wireless Multimedia Communications, Artech House Publishers, London, UK, 1999.

[5] T. Hwang, C. Yang, G. Wu, S. Li, and G. Y. Li, "OFDM and its wireless applications: a survey," IEEE Transactions on Vehicular Technology, vol. 58, no. 4, pp. 1673-1694, 2009.

[6] M. Chiani, M. Z. Win, and A. Zanella, "On the capacity of spatially correlated MIMO Rayleigh-fading channels," IEEE Transactions on Information Theory, vol. 49, no. 10, pp. 23632371, 2003.

[7] A. Abdi and M. Kaveh, "A space-time correlation model for multielement antenna systems in mobile fading channels," IEEE Journal on Selected Areas in Communications, vol. 20, no. 3, pp. 550-560, 2002.

[8] A. F. Molisch, M. Steinbauer, M. Toeltsch, E. Bonek, and R. S. Thomä, "Capacity of MIMO systems based on measured wireless channels," IEEE Journal on Selected Areas in Communications, vol. 20, no. 3, pp. 561-569, 2002.
[9] H. Xu, D. Chizhik, H. Huang, and R. Valenzuela, "A generalized space-time multiple-input multiple-output (MIMO) channel model," IEEE Transactions on Wireless Communications, vol. 3, no. 3, pp. 966-975, 2004.

[10] H. Nishimoto, Y. Ogawa, T. Nishimura, and T. Ohgane, "Measurement-based performance evaluation of MIMO spatial multiplexing in a multipath-rich indoor environment," IEEE Transactions on Antennas and Propagation, vol. 55, no. 12, pp. 3677-3689, 2007.

[11] J. P. Kermoal, L. Schumacher, K. I. Pedersen, P. E. Mogensen, and F. Frederiksen, "A stochastic MIMO radio channel model with experimental validation," IEEE Journal on Selected Areas in Communications, vol. 20, no. 6, pp. 1211-1226, 2002.

[12] W. Weichselberger, M. Herdin, H. Özcelik, and E. Bonek, "A stochastic MIMO channel model with joint correlation of both link ends," IEEE Transactions on Wireless Communications, vol. 5, no. 1, pp. 90-99, 2006.

[13] Q. H. Spencer, B. D. Jeffs, M. A. Jensen, and L. L. Swindlehurst, "Modeling the statistical time and angle of arrival characteristics of an indoor multipath channel," IEEE Journal on Selected Areas in Communications, vol. 18, no. 3, pp. 347-360, 2000.

[14] J. B. Andersen and K. I. Pedersen, "Angle-of-arrival statistics for low resolution antennas," IEEE Transactions on Antennas and Propagation, vol. 50, no. 3, pp. 391-395, 2002.

[15] K. N. Le, "On angle-of-arrival and time-of-arrival statistics of geometric scattering channels," IEEE Transactions on Vehicular Technology, vol. 58, no. 8, pp. 4257-4264, 2009.

[16] S. Kozono and S. Sakagami, "Correlation coefficient on base station diversity for land mobile communication systems," IEICE Communications Society: Transactions on Communications, vol. 70, no. 4, pp. 476-482, 1987. 

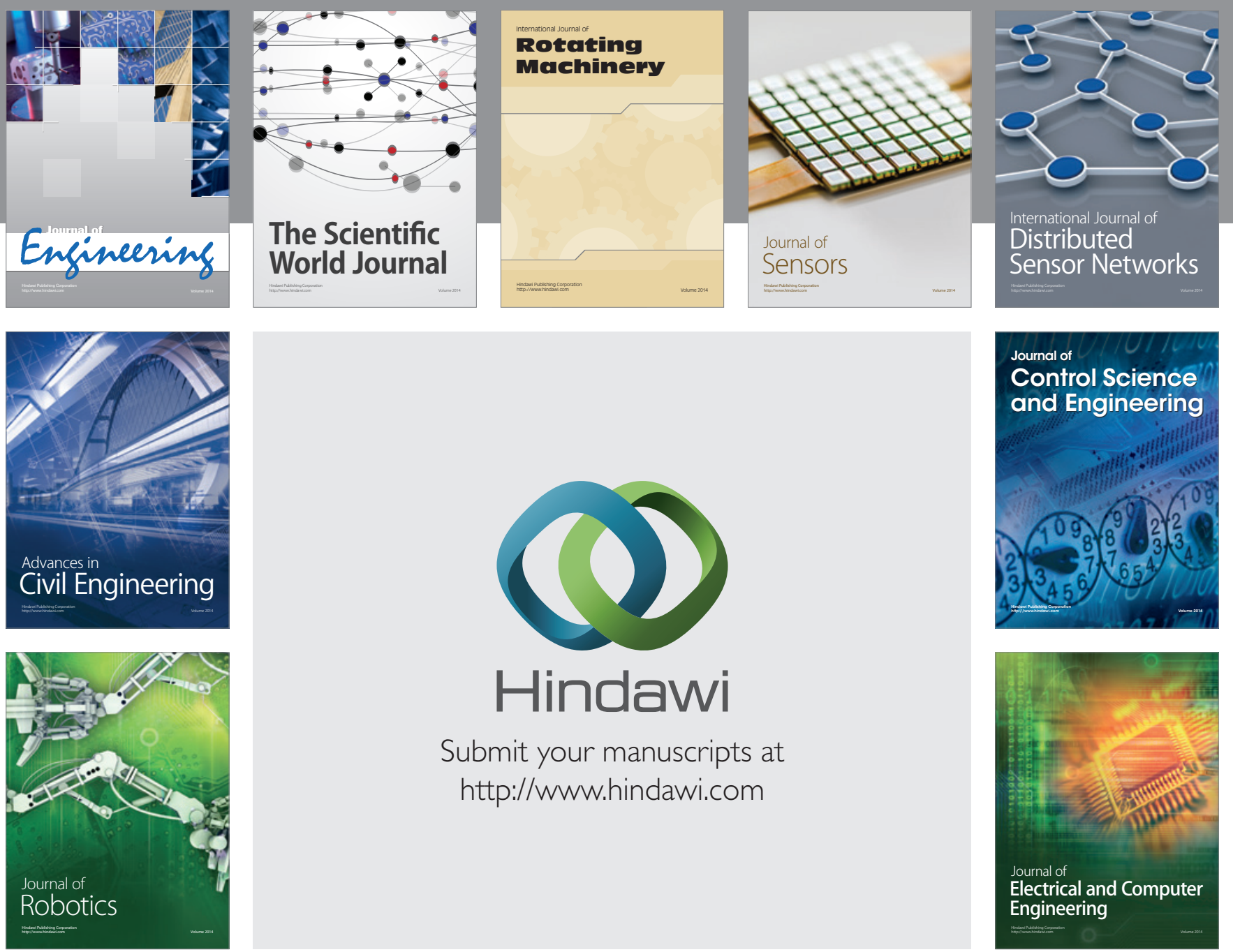

Submit your manuscripts at

http://www.hindawi.com
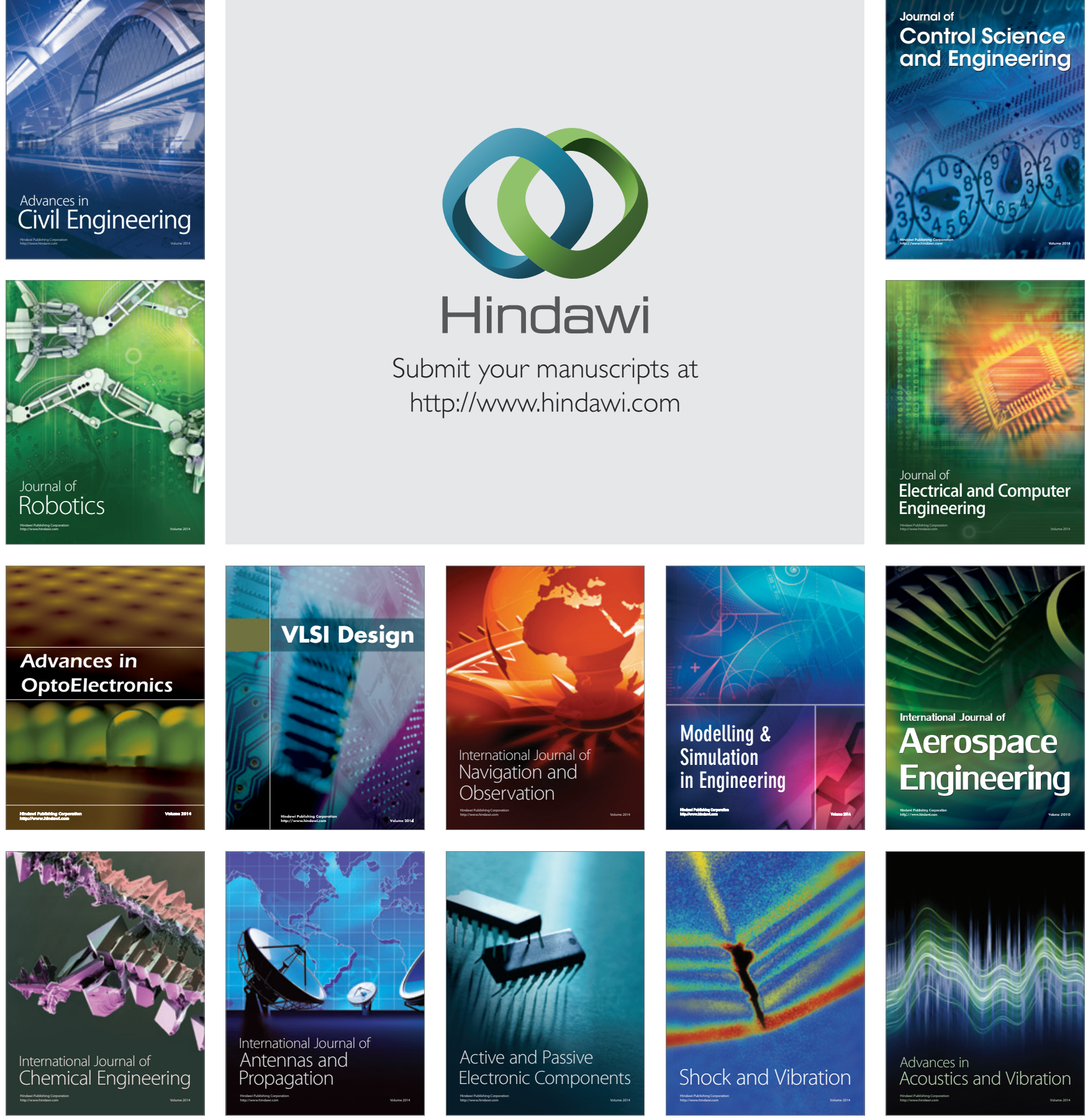